\title{
Reduced-Complexity QAM-Aided Space-Time Shift Keying
}

\author{
Shinya Sugiura, Member, IEEE, Chao Xu, Student Member, IEEE, and Lajos Hanzo, Fellow, IEEE
}

\begin{abstract}
A novel reduced-complexity near-optimal detection algorithm is proposed for enhancing the recent coherently-detected SpaceTime Shift Keying (STSK) scheme employing arbitrary constellations, such as $\mathcal{L}$-point Phase-Shift Keying (PSK) and Quadrature Amplitude Modulation (QAM). The proposed detector relies on a modified Matched Filter (MF) concept. More specifically, we exploit both the constellation diagram of the modulation scheme employed as well as the Inter-ElementInterference (IEI)-free STSK architecture. It is revealed that the proposed detector is capable of approaching the optimal Maximum Likelihood (ML) detector's performance, while avoiding the exhaustive ML search.
\end{abstract}

Index Terms-Diversity and multiplexing tradeoff, space-time shift keying, spatial modulation, matched filter, multiple antenna array.

\section{INTRODUCTION}

The recent Multiple-Input Multiple-Output (MIMO) concept of Spatial Modulation (SM) [1]-[4] or Space-Shift Keying (SSK) [5], [6] is capable of increasing the achievable transmission rate with the aid of multiple-antenna aided systems, which is ensured without resorting to spatial multiplexing [7]. To be more specific, since the SM transmitter activates one out of $M$ antenna elements for conveying additional information bits during each symbol interval, no Inter-Element-Interference (IEI) is imposed on the receiver, hence potentially enabling low-complexity single-stream detection.

Due to the different system architecture of classic spatial multiplexing and of the SM/SSK schemes, new detection algorithms specific to the SM/SSK schemes have been developed, which may be classified into two fundamental categories, namely the low-complexity Matched-Filter (MF) based detector [1] and the single-antenna-based optimal Maximum-Likelihood (ML) detector [2]. In practice, the majority of the previous SM/SSK receivers have adopted the singlestream ML detector [2]-[6], where the optimal BER performance is achieved at the cost of an increased decoding complexity. On the other hand, the MF-based detector [1] exhibits a significantly reduced complexity, since the antenna index $m$ and the modulated constellation point $l$ are separately estimated. However, as mentioned in [2], [8], this sub-optimal detector only works under the idealized assumption of encountering noiseless channels at the antenna-index estimation stage.

Recently, the novel concept of Space-Time Shift Keying (STSK) has been proposed in [9]-[11], where the encoding principle is characterized by the fact that one out of $Q$ space-time dispersion matrices is selected, while the above-mentioned SM and SSK schemes simply activates one out of $M$ antenna elements. Since the STSK scheme is capable of exploiting both the space- and time-dimensions, it allows us to strike a flexible balance between the maximum attainable diversity order and the throughput. ${ }^{1}$ Previous studies of the STSK scheme [9]-[11] also considered the optimal single-stream-based ML detector, similarly to the SM/SSK schemes [2]. One exception is constituted by the solution in [12], where a reduced-complexity detection algorithm was developed in

S. Sugiura is with the Toyota Central Research and Development Laboratories, Inc., Aichi, 480-1192, Japan (e-mail: sugiura@mosk.tytlabs.co.jp).

C. Xu and L. Hanzo are with the School of Electronics and Computer Science, University of Southampton, Southampton, SO17 1BJ, UK (e-mail: $\{$ cx1g08,lh\}@ecs.soton.ac.uk).

${ }^{1}$ In order to expound a little further, both the SM and SSK schemes are subsumed by the STSK arrangement, as demonstrated in [9], [11]. the context of Differentially-encoded STSK (DSTSK) systems [9], which was assisted by Multiple-Symbol Differential Sphere Decoding (MSDSD). However, the applicability of this detector is limited to specific low-order constellations, such as On-Off Keying (OOK), Binary Phase-Shift Keying (BPSK), Quadrature PSK (QPSK) and 8PSK. Regretfully, it is not suitable for bandwidth-efficient Quadrature Amplitude Modulation (QAM) [13].

Against this background, the novel contribution of this paper is that an efficient near-optimal detector is proposed for the STSK scheme. More specifically, the detector relies on a modified MF concept, while taking into account the specific constellation diagram of the modulation scheme employed. The proposed detector is applicable to STSK receiver employing arbitrary modulation schemes, including high-order QAM. Furthermore, since the STSK scheme subsumes the family of SM/SSK schemes as its special case, the proposed lowcomplexity detector is directly applicable to the class of SM/SSK schemes. Finally, the computational complexity imposed by the proposed detector is quantified and compared to that of the conventional detectors in [1] and [2].

The remainder of this paper is organized as follows. Section II outlines the system model of the STSK scheme. In Section III we commence by reviewing the conventional detectors and propose the novel near-optimal reduced-complexity detection scheme, while Section IV provides our simulation results. Finally, Section V concludes this paper.

\section{System Model}

In this section, we briefly review the encoding principle and the received signal model of the STSK scheme [9]. At the STSK transmitter, information bits are encoded with the aid of two different operations, namely the dispersion-matrix activation and the classic $\mathcal{L}$-PSK/QAM modulation. More specifically, the $Q$ spacetime dispersion matrices $\mathbf{A}_{q} \in \mathcal{C}^{M \times T}(q=1, \cdots, Q)$ satisfying the power-constraint relationship of $\operatorname{tr}\left[\mathbf{A}_{q}^{H} \mathbf{A}_{q}\right]=T$ are assigned to the transmitter in advance of transmissions ${ }^{2}$, where $M$ and $T$ denote the number of transmit antennas and the number of symbols per space-time block duration, respectively. Firstly, $B=\log _{2}(Q \cdot \mathcal{L})$ information bits per space-time block are input to the transmitter, and then the input bits are Serial-to-Parallel $(\mathrm{S} / \mathrm{P})$ converted to $B_{1}=\log _{2} Q$ and $B_{2}=\log _{2} \mathcal{L}$ bits. Next, one out of $Q$ dispersion matrices is selected as $\mathbf{A}_{q}$ according to the $B_{1}=\log _{2} Q$ input bits, while the $B_{2}=\log _{2} \mathcal{L}$ input bits are modulated to a PSK/QAM symbol $s_{l}$. Finally, a space-time codeword $\mathbf{S}_{q, l}=s_{l} \mathbf{A}_{q} \in \mathcal{C}^{M \times T}$ is transmitted from the $M$ transmit antenna elements over $T$ symbol durations. Note that, the normalized transmission rate of the STSK scheme is given by $R=\log _{2}(Q \cdot \mathcal{L}) / T$.

The corresponding block-based signals $\mathbf{Y} \in \mathcal{C}^{N \times T}$ received at the

${ }^{2}$ The optimizations of the $Q$ dispersion matrices typically requires a certain cost function and an appropriate search algorithm, which substantially affects the achievable performance of the STSK. Furthermore, depending on the constraint imposed on the dispersion matrices, we may have different STSK classes, such as synchronous STSK, asynchronous STSK and differentiallyencoded STSK, as shown in [9]. However, since the main focus of this paper is the design of a novel detection scheme, rather than the dispersion-matrix optimization, the detailed investigations are left for future study. 
STSK receiver may be expressed as

$$
\mathbf{Y}=\mathbf{H S}+\mathbf{N}
$$

where $\mathbf{H} \in \mathcal{C}^{N \times M}$ represent the channel components, each obeying the complex-valued Gaussian distribution having a zero mean and a unity variance, i.e. $\mathcal{C N}(0,1)$, while each noise element of $\mathbf{N} \in \mathcal{C}^{N \times T}$ is the complex-valued Gaussian variable obeying $\mathcal{C N}\left(0, N_{0}\right)$. Furthermore, $N$ denotes the number of receive antennas and $N_{0}$ represents the noise variance.

By implementing the vectorial stacking operation $\operatorname{vec}(\cdot)$ at both sides of Eq. (1) as shown in [9], we arrive at the equivalent system model as

$$
\overline{\mathbf{Y}}=\overline{\mathbf{H}} \mathbf{K}_{q, l}+\overline{\mathbf{N}}
$$

where we have

$$
\begin{array}{rlrl}
\overline{\mathbf{Y}} & =\operatorname{vec}(\mathbf{Y}) & & \in \mathcal{C}^{N T \times 1} \\
\overline{\mathbf{H}} & =\left[\overline{\mathbf{h}}_{1}, \cdots, \overline{\mathbf{h}}_{Q}\right]=\left(\mathbf{I}_{T} \otimes \mathbf{H}\right) \chi & \in \mathcal{C}^{N T \times Q} \\
\chi & =\left[\operatorname{vec}\left(\mathbf{A}_{1}\right), \cdots, \operatorname{vec}\left(\mathbf{A}_{Q}\right)\right] & & \in \mathcal{C}^{M T \times Q} \\
\overline{\mathbf{N}} & =\operatorname{vec}(\mathbf{N}) & & \in \mathcal{C}^{N T \times 1}
\end{array}
$$

and

$$
\begin{gathered}
\mathbf{K}_{q, l}=\left[0, \cdots, 0, s_{l}, 0, \cdots, 0\right] . \\
q \text { th element }
\end{gathered}
$$

Here, $\otimes$ represents the Kronecker product operation. We note that since the signal vector $\mathbf{K}_{q, l}$ contains only a single non-zero element, the equivalent received signal model of Eq. (2) does not suffer from the effects of IEI.

Furthermore, the receiver structure of Eq. (2) is similar to those of the SM [1], [2] and SSK schemes [5], [6]. More specifically, the SM and SSK schemes may be interpreted as the special case of the STSK arrangement, by setting the STSK parameters to $M=Q, T=1$ and

$$
\mathbf{A}_{1}=\left[\begin{array}{c}
1 \\
0 \\
\vdots \\
0
\end{array}\right], \mathbf{A}_{2}=\left[\begin{array}{c}
0 \\
1 \\
\vdots \\
0
\end{array}\right], \cdots, \mathbf{A}_{Q}=\left[\begin{array}{c}
0 \\
\vdots \\
0 \\
1
\end{array}\right]
$$

Hence, the STSK detector, which will be developed in this paper, is readily applicable to the family of SM, the SSK schemes. Therefore, as shown in [9], the maximum achievable diversity order is $d=N \cdot \min (M, T)$, where $N$ represents the receive diversity while $\min (M, T)$ corresponds to the transmit diversity order. This implies that the SM and SSK schemes, having $T=1$, fail to attain any transmit diversity gain, unlike the STSK scheme.

In the rest of this paper, we employ the parametric notation of 'STSK $(M, N, T, Q)$ ' for the sake of space economy.

\section{LOW-COMLEXITy MF-BASEd STSK DETECTOR}

This section firstly introduces the two conventional detectors in the context of the STSK arrangement, namely the conventional MFbased detector [1] and the single-antennna-based ML detector [2]. Then we continue by outlining the near-optimal receiver architecture advocated, which exploits the properties of the $\mathcal{L}$-PSK/QAM constellations employed. Furthermore, we compare the computational complexity imposed by these three detectors. Note that the aim of the STSK detector is to identify the transmitted index set $(q, l)$ in a reliable and low-complexity manner.

\section{A. The Conventional MF-Based Detector [1]}

In the conventional detector of [1], the Hermitian transpose of the equivalent channel matrix $\overline{\mathbf{H}}$ is multiplied by the equivalent received signal $\overline{\mathbf{Y}}$ in order to formulate the decision metric of $\mathbf{G}=\left[g_{1}, \cdots, g_{Q}\right]^{T} \in \mathcal{C}^{N T \times 1}$, as follows:

$$
\mathbf{G}=\overline{\mathbf{H}}^{H} \overline{\mathbf{Y}} \text {. }
$$

Then, the index $q$ of the activated dispersion matrix and the transmitted symbol index $l$ are estimated separately, as follows:

$$
\begin{aligned}
& \hat{q}=\arg \max _{q}\left|g_{q}\right|, \\
& \hat{l}=\mathcal{D}\left(g_{\hat{q}}\right),
\end{aligned}
$$

where $\mathcal{D}$ denotes the demodulation function. Note that since the transmitted-symbol estimation process of Eq. (11) includes the result of the dispersion-matrix estimation formulated in Eq. (10), the potential mis-detection of $\hat{q}$ may induce error propagation. Importantly, while the search space of this detector determined by Eqs. (10) and $(11)$, is as low as the order of $(Q+\mathcal{L})$, this detection scheme tends to exhibit an error floor in fading environments [2], [8]. This is mainly due to the fact that low-complexity MF-operation of Eq. (9) ignores the effect of the channel's fading envelope, as well as because the decision metric of Eq. (10) only considers the absolute value of the matched-filtered symbol, rather than individually considering each constellation point. In Section III-C below, our detector will be further developed for the sake of combating these limitations, hence ultimately achieving a higher BER performance than that of the detector of this section.

\section{B. The Single-Stream-Based ML Detector [2], [9]}

The optimal ML performance may be attained by implementing exhaustive search over the legitimate STSK codewords $\mathbf{K}_{q, l}(1 \leq$ $q \leq Q, 1 \leq l \leq \mathcal{L})$ at the cost of an increased complexity. To be more specific, by maximizing the probability of $P\left(\overline{\mathbf{Y}} \mid \overline{\mathbf{H}}, \mathbf{K}_{q, l}\right)$, the ML search may be expressed as

$$
\begin{aligned}
(\hat{q}, \hat{l}) & =\arg \min _{q, l}\left\|\overline{\mathbf{Y}}-\overline{\mathbf{H}} \mathbf{K}_{q, l}\right\|^{2} \\
& =\arg \min _{q, l}\left\|\overline{\mathbf{Y}}-s_{l} \overline{\mathbf{h}}_{q}\right\|^{2},
\end{aligned}
$$

where $\overline{\mathbf{h}}_{q}$ is the $q$ th column of $\overline{\mathbf{H}}$. As shown in Eq. (13), the search space size of the ML detector is the order of $(Q \cdot \mathcal{L})$, which is higher than $(Q+\mathcal{L})$ in the detector [1] outlined in Section III-A. Nevertheless, it should be emphasized that this optimal ML STSK detection scheme typically imposes a lower complexity in comparison to that of BLAST-style spatial multiplexing systems, since Eq. (13) is free from the effects of IEI, which is the explicit benefit of the STSK philosophy [9].

\section{The proposed MF-Based Detector}

In this section, we present the new reduced-complexity STSK detector. Let us first define the modified equivalent channels $\mathbf{H}^{\prime}$ as

$$
\mathbf{H}^{\prime}=\left[\frac{\overline{\mathbf{h}}_{1}}{\left\|\overline{\mathbf{h}}_{1}\right\|}, \cdots, \frac{\overline{\mathbf{h}}_{Q}}{\left\|\overline{\mathbf{h}}_{Q}\right\|}\right] .
$$

Then, we have the MF output of

$$
\mathbf{Z}=\left[z_{1}, \cdots, z_{Q}\right]^{T}=\mathbf{H}^{\prime H} \overline{\mathbf{Y}} .
$$

Next, we consider $V$ number of unit-norm vectors $\mathbf{x}_{v}(v=$ $1, \cdots, V)$, which are specific to the PSK/QAM modulation scheme employed. More explicitly, as exemplified in Fig. 2 for 16-QAM, we draw a straight line from the origin to each constellation point, 
TABLE I

MAPPING EXAMPLeS FROM Modulation SCHEMES to Points $\mathbf{x}_{v}(v=1, \cdots, V)$.

\begin{tabular}{|c|c|c|c|c|c|c|}
\hline $\begin{array}{l}\text { Modulation } \\
\text { Scheme }\end{array}$ & \multicolumn{3}{|c|}{$\mathbf{x}_{v}(v=1, \cdots, V)$} & $\begin{array}{l}\text { Modulation } \\
\text { Scheme }\end{array}$ & \multicolumn{2}{|c|}{$\mathbf{x}_{v}(v=1, \cdots, V)$} \\
\hline BPSK & $\overline{\mathbf{x}_{1}}$ & $=$ & {$[1,0]$} & \multirow[t]{12}{*}{ 64-QAM } & $\mathbf{x}_{3}$ & \multirow{3}{*}{$\begin{array}{l}=\left[\frac{3}{\sqrt{10}}, \frac{1}{\sqrt{10}}\right. \\
=\quad\left[\frac{7}{\sqrt{58}}, \frac{3}{\sqrt{58}}\right.\end{array}$} \\
\hline QPSK & $\mathbf{x}_{1}$ & $=$ & {$[1,0]$} & & $\mathbf{x}_{4}$ & \\
\hline & $\mathbf{x}_{2}$ & $=$ & {$[0,1]$} & & $\mathbf{x}_{5}$ & \\
\hline \multirow[t]{3}{*}{ 8-PSK } & $\overline{\mathbf{x}_{1}}$ & $=$ & {$[1,0]$} & & $\mathbf{x}_{6}$ & \multirow{3}{*}{$\begin{array}{l}=\left[\frac{\sqrt{34}}{7}, \frac{\sqrt{34}}{\sqrt{74}}, \frac{5}{\sqrt{74}}\right] \\
=\left[\frac{1}{\sqrt{2}}, \frac{1}{\sqrt{2}}\right] \\
=\left[\frac{5}{\sqrt{74}}, \frac{7}{\sqrt{74}}\right]\end{array}$} \\
\hline & $\mathbf{x}_{2}$ & & {$\left[\frac{1}{\sqrt{2}}, \frac{1}{\sqrt{2}}\right]$} & & & \\
\hline & $\mathbf{x}_{3}$ & $=$ & {$[0,1]$} & & $\mathbf{x}_{8}$ & \\
\hline 4-QAM & $\mathbf{x}_{1}$ & $=$ & {$\left[\frac{1}{\sqrt{2}}, \frac{1}{\sqrt{2}}\right]$} & & $\mathbf{x}_{9}$ & \multirow{4}{*}{$\begin{array}{l}=\left[\frac{3}{\sqrt{34}}, \frac{5}{\sqrt{34}}\right] \\
=\left[\frac{3}{\sqrt{58}}, \frac{7}{\sqrt{58}}\right] \\
=\left[\frac{1}{\sqrt{10}}, \frac{3}{\sqrt{10}}\right] \\
=\left[\frac{1}{\sqrt{26}}, \frac{5}{\sqrt{26}}\right]\end{array}$} \\
\hline \multirow[t]{3}{*}{ 16-QAM } & $\mathbf{x}_{1}$ & & {$\left[\frac{3}{\sqrt{10}}, \frac{1}{\sqrt{10}}\right]$} & & $\mathbf{x}_{10}$ & \\
\hline & $\mathbf{x}_{2}$ & & {$\left[\frac{1}{\sqrt{2}}, \frac{1}{\sqrt{2}}\right]$} & & $\mathbf{x}_{11}$ & \\
\hline & $\mathbf{x}_{3}$ & & {$\left[\frac{1}{\sqrt{10}}, \frac{3}{\sqrt{10}}\right]$} & & $\mathbf{x}_{12}$ & \\
\hline \multirow[t]{2}{*}{ 64-QAM } & $\mathbf{x}_{1}$ & & {$\left[\frac{1}{\sqrt{50}}, \frac{1}{\sqrt{50}}\right]$} & & \multirow[t]{2}{*}{$\mathbf{x}_{13}$} & \multirow[t]{2}{*}{$=\left[\frac{1}{\sqrt{50}}, \frac{7}{\sqrt{50}}\right.$} \\
\hline & $\mathbf{x}_{2}$ & & {$\left[\frac{5}{\sqrt{26}}, \frac{1}{\sqrt{26}}\right]$} & & & \\
\hline
\end{tabular}



Fig. 1. The proposed detector structure.

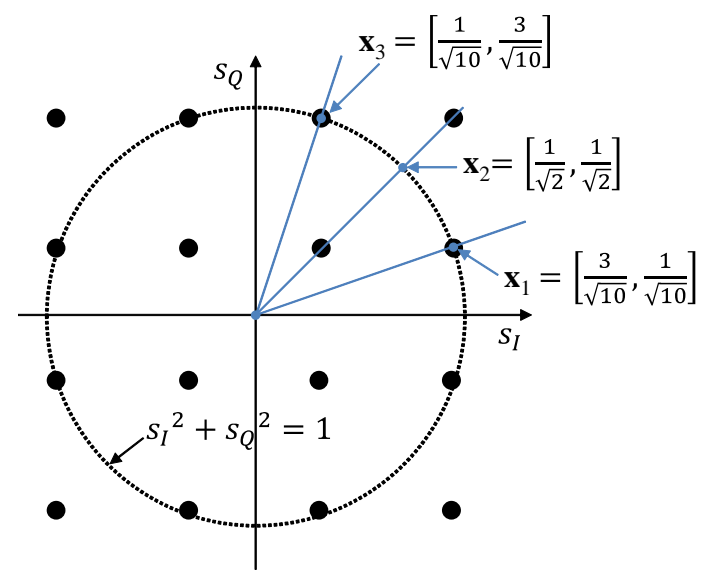

Fig. 2. Calculation of $\mathbf{X}=\left[\mathbf{x}_{1}^{T}, \cdots, \mathbf{x}_{V}^{T}\right]$ for 16-QAM.

which is located in the first quadrant. Then, we have $V$ points $\mathbf{x}_{v}=\left[\begin{array}{ll}x_{v, I} & x_{v, Q}\end{array}\right](v=1, \cdots, V)$, which are given by the crossing points of the above-mentioned $V$ lines and the unit circle of $s_{I}^{2}+s_{Q}^{2}=1 .^{3}$ For more detail, we listed in Table I a set of examples for characterizing the mapping of classic PSK/QAM symbols to the

${ }^{3}$ To elaborate a little further, this process of generating $\mathbf{x}_{v},(v=1, \cdots, V)$ from the constellation points is applicable to modulation schemes, which have symmetric properties around both the $s_{I}$ and $s_{Q}$ axes. This indicates that the proposed detection may be used for most of the conventional PSK/QAM schemes. For other constellation schemes, which do not have such symmetric properties, all the constellation points have to be considered for the calculation of $\mathbf{x}_{v}(v=1, \cdots, V)$. Naturally, this is only possible at the cost of an increased value of $V$, hence an increased decoding complexity, as formulated in Eq. (29) of Section III-D. corresponding sets of $\mathbf{x}_{v}(v=1, \cdots, V)$.

Now, we have the decision metric of $\mathbf{G}^{\prime} \in \mathcal{R}^{Q \times V}$

$$
\mathbf{G}^{\prime}=\mathbf{P X}
$$

where we have

$$
\begin{aligned}
& \mathbf{P}=[|\Re(\mathbf{Z})||\Im(\mathbf{Z})|] \\
& \mathbf{X}=\left[\mathbf{x}_{1}^{T}, \cdots, \mathbf{x}_{V}^{T}\right]
\end{aligned}
$$

while $\Re(\cdot)$ and $\Im(\cdot)$ represent the real and imaginary parts of a matrix, respectively. ${ }^{4}$ Finally, the transmitted dispersion-matrix index $q$ and symbol index $l$ are estimated according to

$$
\begin{aligned}
(\hat{q}, \hat{v}) & =\arg \max _{q, v}\left(g_{q, v}^{\prime}\right), \\
\hat{l} & =\mathcal{D}\left(z_{\hat{q}}\right),
\end{aligned}
$$

where $g_{q, v}^{\prime}$ is defined as the $q$ th-row and the $v$ th-column element of $\mathbf{G}^{\prime}$.

We note, furthermore, that the classic MF detector tends to exhibit an error floor in joint Multi-User Detection (MUD) scenarios of Code-Division Multiple Access (CDMA) systems [14], which is also the case for spatial multiplexing systems. This is because the MF operation is incapable of suppressing the inter-user or inter-element interferences. On the other hand, as a benefit of avoiding the IEI in our STSK scheme, the modified MF operation of Eq. (15) as well as the corresponding decision-metric generation of Eq. (16) potentially facilitates a simple solution for the STSK codeword detection, without requiring elaborate interference cancellation.

To expound a little futher, the estimation of the dispersion-matrix index $q$, which is expressed in Eq. (19), takes into account the specifics of the constellation employed, while the original detection metric of Eq. (10) does not. More specifically, the generation of the decision metric $\mathbf{G}^{\prime}$ represented by Eq. (16) reflects the ratio of each constellation point's real value over the corresponding imaginary value. To be more specific, given a transmitted index set of $(q, l)$ and the corresponding value of $v$, the correct element $g_{q, v}^{\prime}$ of the decision

${ }^{4}$ It is specific for the OOK scenario of $\mathcal{L}=1$ that $\mathbf{P}=[\Re(\mathbf{Z}) \Im(\mathbf{Z})]$ may be employed instead of Eq. (17), which would result in a slightly better detection performance. 
metric $\mathbf{G}^{\prime}$ is represented by

$$
\begin{aligned}
g_{q, v}^{\prime} & =x_{v, I}\left|\left\|\overline{\mathbf{h}}_{q}\right\| \Re\left(s_{l}\right)+\Re\left(\frac{\overline{\mathbf{h}}_{q}^{H}}{\left\|\overline{\mathbf{h}}_{q}\right\|} \overline{\mathbf{N}}\right)\right| \\
& +x_{v, Q}\left|\left\|\overline{\mathbf{h}}_{q}\right\| \Im\left(s_{l}\right)+\Im\left(\frac{\overline{\mathbf{h}}_{q}^{H}}{\left\|\overline{\mathbf{h}}_{q}\right\|} \overline{\mathbf{N}}\right)\right|,
\end{aligned}
$$

while the incorrect element $g_{\tilde{q}, v^{\prime}}^{\prime}\left(\tilde{q} \neq q, 1 \leq v^{\prime} \leq V\right)$ may be expressed as

$$
\begin{aligned}
g_{\tilde{q}, v^{\prime}}^{\prime} & =x_{v^{\prime}, I}\left|\Re\left(\frac{\overline{\mathbf{h}}_{\tilde{q}}^{H}}{\left\|\overline{\mathbf{h}}_{\tilde{q}}\right\|} \overline{\mathbf{h}}_{q} s_{l}\right)+\Re\left(\frac{\overline{\mathbf{h}}_{\tilde{q}}^{H}}{\left\|\overline{\mathbf{h}}_{\tilde{q}}\right\|} \overline{\mathbf{N}}\right)\right| \\
& +x_{v^{\prime}, Q}\left|\Im\left(\frac{\overline{\mathbf{h}}_{\tilde{q}}^{H}}{\left\|\overline{\mathbf{h}}_{\tilde{q}}\right\|} \overline{\mathbf{h}}_{q} s_{l}\right)+\Im\left(\frac{\overline{\mathbf{h}}_{\tilde{q}}^{H}}{\left\|\overline{\mathbf{h}}_{\tilde{q}}\right\|} \overline{\mathbf{N}}\right)\right|
\end{aligned}
$$

Provided that we have

$$
g_{q, v}^{\prime}>g_{\tilde{q}, v^{\prime}}^{\prime} \quad\left(q \neq \tilde{q}, 1 \leq v^{\prime} \leq V\right)
$$

the correct index $q$ is found during each block interval. Here, let us consider the ultimate scenario of $N_{0} \rightarrow 0$. Then Eq. (23) becomes

$$
\begin{aligned}
& \left\|\overline{\mathbf{h}}_{q}\right\| \underbrace{\left\{x_{v, I}\left|\Re\left(s_{l}\right)\right|+x_{v, Q}\left|\Im\left(s_{l}\right)\right|\right\}}_{\phi}> \\
& x_{v^{\prime}, I}\left|\Re\left(\frac{\overline{\mathbf{h}}_{\tilde{q}}^{H}}{\left\|\overline{\mathbf{h}}_{\tilde{q}}\right\|} \overline{\mathbf{h}}_{q} s_{l}\right)\right|+x_{v^{\prime}, Q} \mid \Im\left(\frac{\overline{\mathbf{h}}_{\tilde{q}}^{H}}{\left.\| \overline{\mathbf{h}}_{\tilde{q} \|} \overline{\mathbf{h}}_{q} s_{l}\right) \mid .}\right.
\end{aligned}
$$

We note that considering the relationships of $\left|x_{v^{\prime}, I}\right|^{2}+\left|x_{v^{\prime}, Q}\right|^{2}=1$ as well as of $\left|\Re\left(s_{l}\right)\right|,\left|\Im\left(s_{l}\right)\right| \geq 0, \phi=x_{v, I}\left|\Re\left(s_{l}\right)\right|+x_{v, Q}\left|\Im\left(s_{l}\right)\right|$ of Eq. (24) may be upper-bounded by

$$
\begin{aligned}
\phi & =x_{v, I}\left|\Re\left(s_{l}\right)\right|+x_{v, Q}\left|\Im\left(s_{l}\right)\right| \\
& \leq \sqrt{\left|\Re\left(s_{l}\right)\right|^{2}+\left|\Im\left(s_{l}\right)\right|^{2}}=\left|s_{l}\right| .
\end{aligned}
$$

In Eq. (25) we have equality if and only if

$$
x_{v, I} / x_{v, Q}=\left|\Re\left(s_{l}\right)\right| /\left|\Im\left(s_{l}\right)\right| .
$$

Therefore, the projection operation from the constellation points $s_{l}(1 \leq l \leq \mathcal{L})$ to the corresponding points $\mathbf{x}_{v}(1 \leq v \leq V)$ on the unit circle, as shown in Fig. 2, is aimed for having $\mathbf{x}_{v}$, which satisfies Eq. (26), hence maximizes the left hand side of Eq. (24). As a result, Eq. (24) is always satisfied and the conventional detector's error floor can be eliminated.

\section{Complexity Analysis}

In this section, we compare the computational complexity imposed by the three detectors presented in Section III, i.e. the original MFbased detector [1], the optimal ML detector [2] and the proposed detector of Section III-C. Here, we quantified the complexity as the number of real-valued multiplications, where a single complexvalued multiplication is deemed to be equivalent to four real-valued multiplications.

The corresponding complexity per bit may be expressed, respectively, as

$$
\begin{aligned}
C_{\text {Mesleh }} & =\frac{(4 M N T Q+2 N T) / \tau+4 N T Q+2 \mathcal{L}}{\log _{2}(Q \cdot \mathcal{L})}, \\
C_{\mathrm{ML}} & =\frac{(4 M N T Q+4 \mathcal{L} N T Q) / \tau+2 \mathcal{L} N T Q}{\log _{2}(Q \cdot \mathcal{L})},
\end{aligned}
$$

and

$$
C_{\mathrm{MF}}=\frac{(4 M N T Q+3 N T Q) / \tau+4 N T Q+2 V Q+2 \mathcal{L}}{\log _{2}(Q \cdot \mathcal{L})} .
$$

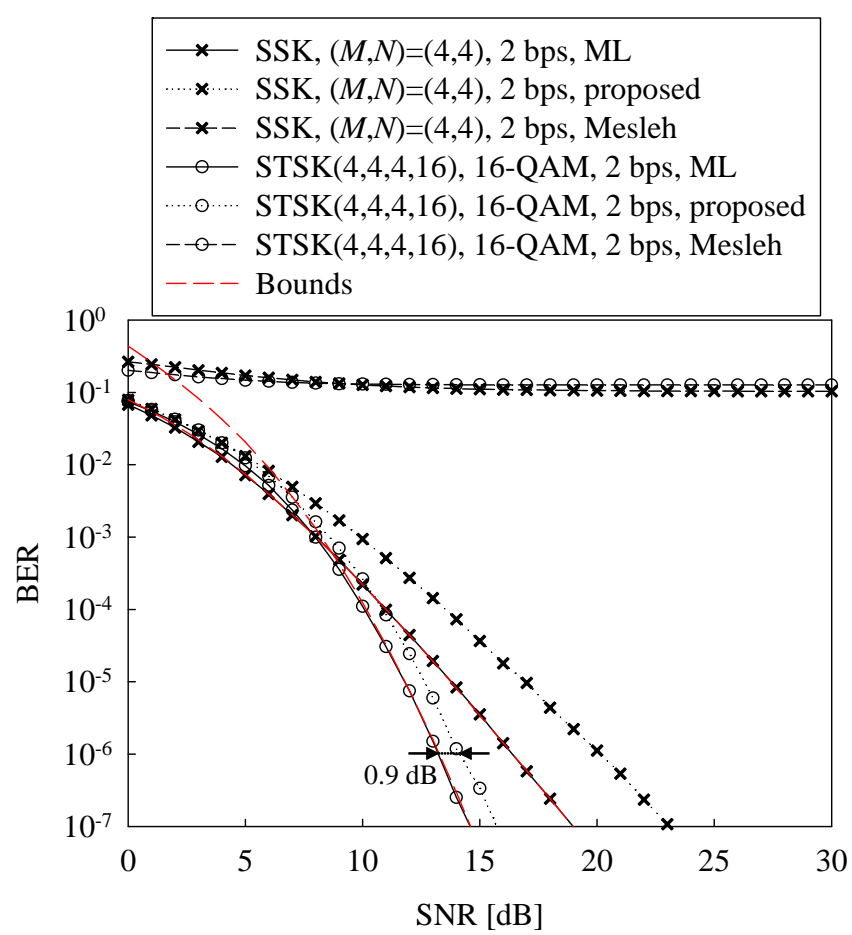

Fig. 3. Achievable BER performance of the 16-QAM modulated STSK $(4,4,4,16)$ scheme and the SSK scheme employing $M=N=4$ transmit and receive antennas, where the optimal ML detector, the proposed MF-based detector and Mesleh's MF-based detector were considered. Here, all the arrangements have the throughput of 2 bits/symbol and are equipped with $M=N=4$ transmit and receive antennas.

Here, the coherence block interval $\tau$ is defined as the number of space-time blocks, where the channel matrix $\mathbf{H}$ remains constant. This indicates that for example $\tau=1$ represents an instantaneously fading scenario and that upon increasing the coherence interval $\tau$, the associated complexity of Eqs. (27)-(29) may decrease, because for example the calculations of $\overline{\mathbf{H}}, s_{l} \overline{\mathbf{h}}_{q},\left\|\overline{\mathbf{h}}_{q}\right\|^{2}$ and $\mathbf{H}^{\prime}$ can be reused.

\section{Performance Results}

In this section we provide our performance results for characterizing both the achievable BER and the computational complexity of the above-mentioned three detectors developed for the STSK scheme.

Figs. 3 and 4 compare the BER of the STSK family, namely of the SM, the SSK and the STSK schemes, employing $M=4$ transmit and $N=4$ receive antenna elements. In Fig. 3 we considered the SSK and the 16-QAM assisted $\operatorname{STSK}(4,4,4,16)$ schemes exhibiting a normalized throughput of $R=2$ bits/symbol, while in Fig. 4 the BER curves of the BPSK-modulated SM and the 8-PSK modulated $\operatorname{STSK}(4,4,2,8)$ schemes having $R=3$ bits/symbol were portrayed. Here, we also plotted the corresponding tight BER upper-bound curves calculated based on the Moment-Generating Function (MGF) [15], in order to confirm the ML detector's BER results.

Observe in both Figs. 3 and 4 that while the original MF detector exhibited an error floors as predicted from [2], [8], the proposed detector achieved a near-optimal performance, which was close to the BER curves of the ML detector. More specifically, the STSK scheme's SNR differences recorded at BER $=10^{-6}$ between the ML detector and the proposed near-optimal detector in Figs. 3 and 4 were as low as $0.9 \mathrm{~dB}$ and $0.5 \mathrm{~dB}$, respectively. Furthermore, based on our extensive simulations, it was found that the performance difference between the optimal ML detector and the proposed MFbased detector was reduced for a higher $N T$ scenario, given a 


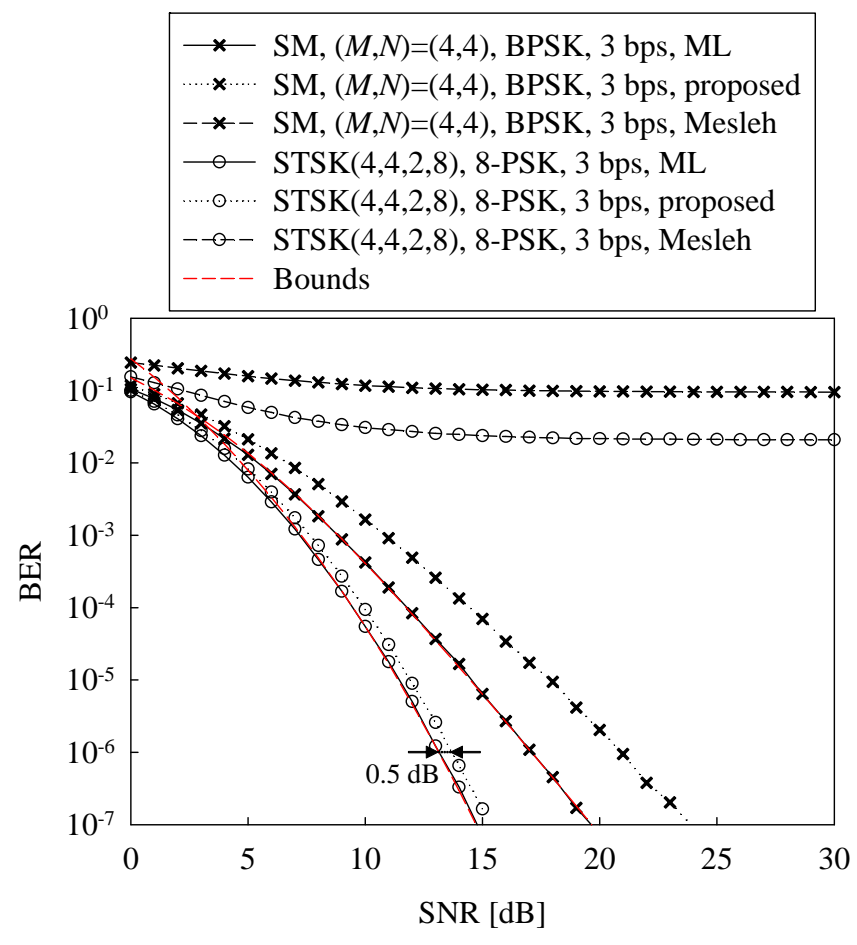

Fig. 4. Achievable BER performance of the 8-PSK modulated STSK $(4,4,2,8)$ scheme and the BPSK-modulated SM scheme employing $M=N=4$ transmit and receive antennas, where the optimal ML detector, the proposed MF-based detector and Mesleh's MF-based detector were considered. Here, all the arrangements have the throughput of 3 bits/symbol and are equipped with $M=N=4$ transmit and receive antennas.

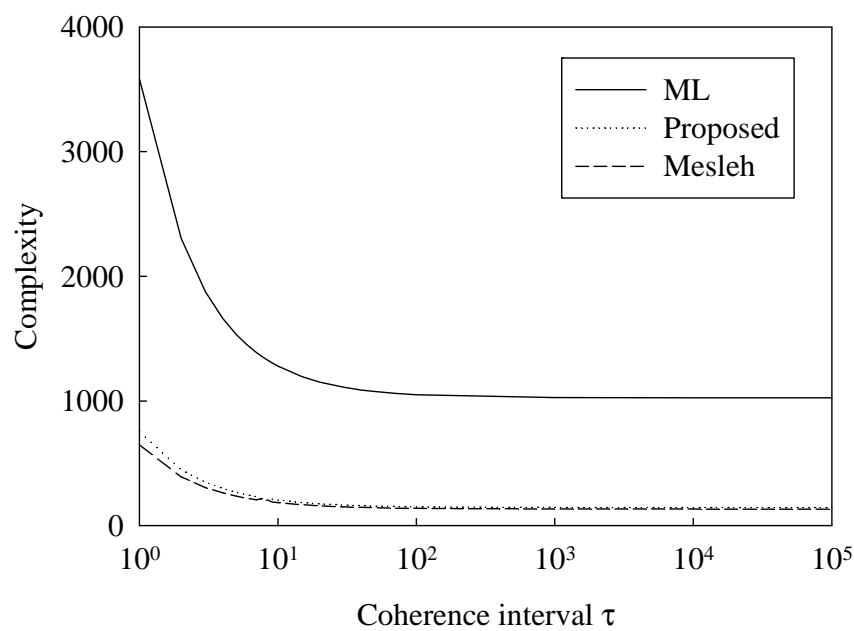

Fig. 5. Complexity comparison between the three detectors, which are presented in Section III, for the 16-QAM assisted $\operatorname{STSK}(4,4,4,16)$ arrangement.

certain constellation scheme. This implies that the employment of the proposed detector may be especially beneficial for the STSK scheme, rather than for the SM and SSK schemes, since the latter two have $T=1$.

Moreover, Fig. 5 shows the computational complexity imposed by the above-mentioned three detectors for the 16-QAM assisted $\operatorname{STSK}(4,4,4,16)$ scheme of Fig. 3. Observe in Fig. 5 that upon increasing the coherence interval $\tau$, the complexity of each detector was reduced towards a certain minimum value. It was found that our
MF-based detector attained as low a complexity as the original MF detector, which is substantially lower than that of the ML detector.

\section{Conclusions}

In this paper, we proposed a reduced-complexity near-optimal detector for the STSK scheme employing an arbitrary PSK/QAM constellation, which exploits the STSK-specific IEI-free system model, rather than that of spatial multiplexing. More specifically, the proposed MF detector takes into account the specific constellation diagram considered. As a result, our detector is capable of achieving a lower complexity than that of ML detection, while avoiding any substantial BER performance loss. Therefore, the employment of this detector further augments the benefits of the STSK scheme.

The proposed detector designed for the class of co-located STSK schemes readily lends itself to cooperative communications [16] as well as relying on semi-blind joint channel estimation and data detection [10].

\section{REFERENCES}

[1] R. Y. Mesleh, H. Haas, S. Sinanovic, C. Ahn, and S. Yun, "Spatial modulation," IEEE Transactions on Vehicular Technology, vol. 57, no. 4, pp. 2228-2242, 2008.

[2] J. Jeganathan, A. Ghrayeb, and L. Szczecinski, "Spatial modulation: optimal detection and performance analysis," IEEE Communications Letters, vol. 12, no. 8, pp. 545-547, 2008.

[3] N. Serafimovski, M. Di Renzo, S. Sinanovic, R. Y. Mesleh, and H. Haas, "Fractional bit encoded spatial modulation (FBE-SM)," IEEE Communications Letters, vol. 14, no. 5, pp. 429-431, 2010.

[4] R. Y. Mesleh, M. Renzo, H. Haas, and P. Grant, "Trellis coded spatial modulation," IEEE Transactions on Wireless Communications, vol. 9, no. 7 , pp. 2349-2361, 2010.

[5] J. Jeganathan, A. Ghrayeb, L. Szczecinski, and A. Ceron, "Space shift keying modulation for MIMO channels," IEEE Transactions on Wireless Communications, vol. 8, no. 7, pp. 3692-3703, 2009.

[6] M. Di Renzo and H. Haas, "A general framework for performance analysis of space shift keying (SSK) modulation for MISO correlated Nakagami-m fading channels," IEEE Transactions on Communications, vol. 58, no. 9, pp. $2590-2603,2010$.

[7] P. Wolniansky, G. Foschini, G. Golden, and R. Valenzuela, "V-BLAST: An architecture for realizing very high data rates over the rich-scattering wireless channel," in Proceedings of the International Symposium on Signals, Systems, and Electronics (ISSSE'98), Pisa, Italy, 1998, pp. 295300.

[8] J. Jeganathan, A. Ghrayeb, and L. Szczecinski, "Generalized space shift keying modulation for MIMO channels," in IEEE 19th International Symposium on Personal, Indoor and Mobile Radio Communications (PIMRC 2008), Cannes, France, 15-18 September 2008, pp. 1-5.

[9] S. Sugiura, S. Chen, and L. Hanzo, "Coherent and differential spacetime shift keying: A dispersion matrix approach," IEEE Transactions on Communications, vol. 58, no. 11, pp. 3219-3230, November 2010.

[10] S. Chen, S. Sugiura, and L. Hanzo, "Semi-blind joint channel estimation and data detection for space-time shift keying systems," IEEE Signal Processing Letters, vol. 17, no. 12, pp. 993 -996, December 2010.

[11] S. Sugiura, S. Chen, and L. Hanzo, "Generalized space-time shift keying designed for flexible diversity-, multiplexing- and complexity-tradeoffs," IEEE Transactions on Wireless Communications, vol. 10, pp. 1-10, 2011, in press (early access at IEEE Xplore).

[12] C. Xu, S. Sugiura, S. X. Ng, and L. Hanzo, "Reduced-complexity noncoherently detected differential space-time shift keying," IEEE Signal Processing Letters, vol. 18, no. 3, pp. 153-156, Mar. 2011.

[13] L. Hanzo, S. Ng, W. T. Webb, and T. Keller, Quadrature amplitude modulation: From basics to adaptive trellis-coded, turbo-equalised and space-time coded OFDM, CDMA and MC-CDMA systems. John Wiley and IEEE Press, 2004, 1036 pages.

[14] L. Hanzo, L.-L. Yang, E.-L. Kuan, and K. Yen, Single and Multi-Carrier CDMA: Multi-User Detection, Space-Time Spreading, Synchronisation and Standards. John Wiley and IEEE Press, 2003, 1060 pages.

[15] M. K. Simon and M. S. Alouini, Digital communication over fading channels. Wiley-IEEE Press, 2005.

[16] S. Sugiura, S. Chen, H. Haas, P. M. Grant, and L. Hanzo, "Coherent versus non-coherent decode-and-forward relaying aided distributed spacetime shift keying," IEEE Transactions on Communications, vol. 59, pp. $1-13,2011$, in press. 